\title{
ON ERROR BOUNDS FOR APPROXIMATIONS TO AGGREGATE CLAIMS DISTRIBUTIONS
}

\author{
BY JAN DHAENE \\ Katholieke Universiteit Leuven, Belgium \\ BJøRn SUNDT \\ University of Bergen, Norway
}

\begin{abstract}
In the present paper we discuss error bounds for approximations to aggregate claims distributions. We consider approximations to convolutions by approximating each of the distributions and taking the convolution of these approximations. For compound distributions we consider two classes of approximations. In the first class we approximate the counting distribution, but keep the severity distribution unchanged, whereas in the second class we approximate the severity distribution, but keep the counting distribution unchanged. We finally look at some examples.
\end{abstract}

\section{INTRODUCTION}

During the last two decades there has developed a large literature on approximations to aggregate claims distributions and related functions, in particular their stop loss transforms. In the present paper we give bounds for some measures of errors caused by such approximations. These measures can also be applied as measures for the distance between two distributions.

In Section 2 we introduce some notation and conventions, and in a short Section 3 we present some simple inequalities for error bounds.

Approximations to convolutions of distributions is the topic of Section 4 . We approximate a convolution by approximating each of the distributions in the convolution and then taking the convolution of the approximations.

Approximations to compound distributions is the topic of Section 5 . We consider two classes of approximations. In the first class we approximate the counting distribution, but keep the severity distribution unchanged, whereas in the second class we approximate the severity distribution, but keep the counting distribution unchanged. Error bounds for approximations where both the counting distribution and the severity distribution are approximated, can be found by application of triangle inequalities.

In Section 6 we finally consider some applications. Further applications of results from the present paper are given in Dhaene \& Sundt (1996). 
The main topic of the present paper is approximations to probability distributions. These approximations are not necessarily distributions themselves. Sometimes one would apply an approximation that could be naturally split into more than one step, e.g. approximating a compound distribution by first approximating its counting distribution and then its severity distribution. In this situation one could first give bounds for the approximation error of the approximation with correct severity distribution and approximated counting distribution, then for the final approximation considered as an approximation to this intermediary approximation, and finally use triangle inequalities to assess the approximation error of the aggregate approximation. In such a procedure, the intermediary approximation would not necessarily be a distribution, and thus in our frame-work it is also of interest to discuss approximations to functions. On this background we have sometimes in our results assumed that the quantity to be approximated is a more general function than a probability distribution. Such generalisations are also possible in some of the other results where we for simplicity have made more restrictive assumptions.

\section{NOTATION AND CONVENTIONS}

In the present paper we shall be concerned with probability distributions on the non-negative integers. We shall approximate such distributions by approximating their discrete densities. Thus we identify a distribution by its discrete density, and for convenience we shall usually mean its discrete density when we talk about a distribution.

Let $\mathcal{P}$ denote the class of (discrete densities of) probability distributions on the non-negative integers. When discussing approximations to compound distributions, we shall restrict the severity distribution to the positive integers, and we therefore also introduce $\mathcal{P}_{+}$as the class of distributions on the positive integers. As we shall approximate distributions in $\mathcal{P}$ and $\mathcal{P}+$ by functions which are not necessarily distributions themselves, we shall also need the classes $\mathcal{F}$ and $\mathcal{F}_{+}$, being respectively the class of functions on the non-negative integers and the class of functions on the positive integers. We see that $\mathcal{P}_{+} \subset \mathcal{P} \subset \mathcal{F}$ and $\mathcal{P}+\subset \mathcal{F}+\subset \mathcal{F}$.

For a function $f \in \mathcal{F}$ we introduce

$$
\begin{gathered}
\mu_{i}(f)=\sum_{x=0}^{\infty} x^{i} f(x) \quad(j=0,1) \\
\Gamma_{f}(x)=\sum_{y=0}^{x} f(y) \quad \Pi_{f}(x)=\sum_{y=x+1}^{\infty}(y-x) f(y) . \quad(x=0,1,2, \ldots)
\end{gathered}
$$

When the quantities $\mu_{0}(f)$ and $\mu_{1}(f)$ appear, it will always be silently assumed that they exist and are finite. When $\Pi_{f}(x)$ appears, it is assumed that $\mu_{0}(f)$ and $\mu_{1}(f)$ converge so that $\Pi_{f}(x)$ is well defined and has a finite value.

If $f \in \mathcal{P}$, then $\Gamma_{f}$ is the corresponding cumulative distribution, $\Pi_{f}$ the stop loss transform, $\mu_{1}(f)$ the mean, and $\mu_{0}(f)=1$. 
As the main purpose of this paper is to study the approximation error for approximations to a distribution, we introduce the following measures for the distance between two functions $f, g \in \mathcal{F}$ :

$$
\begin{gathered}
\varepsilon_{j}(f, g)=\sum_{x=0}^{\infty} x^{j}|f(x)-g(x)| \quad(j=0,1) \\
\eta(f, g)=\sup _{x \geq 0}\left|\Pi_{f}(x)-\Pi_{g}(x)\right| .
\end{gathered}
$$

For evaluating the quality of an approximation only considered as an approximation to the discrete density, $\varepsilon_{0}(f, g)$ is a natural measure for the approximation error. If we want to evaluate the corresponding approximation to the stop loss transform, then $\eta(f, g)$ is a natural measure. We see that $\varepsilon_{0}(f, g)$, $\varepsilon_{1}(f, g)$, and $\eta(f, g)$ are equal to zero if and only if $f=g$

By the notation $x_{+}$we shall mean the maximum of $x$ and zero.

We denote by $I$ the indicator function defined by $I(A)=1$ if the condition $A$ is true and $I(A)=0$ if it is false.

We shall interpret $\Sigma_{i=a}^{b} v_{i}=0$ and $\Pi_{i=a}^{b} v_{i}=1$ when $b<a$.

\section{SOME USEFUL INEQUALITIES}

The following lemma gives some useful inequalities that we shall need later.

Lemma 3.1 Forf, $g, h \in \mathcal{F}$ and $j=0,1$, we have

$$
\begin{gathered}
\varepsilon_{j}(f, g) \leq \varepsilon_{j}(f, h)+\varepsilon_{j}(h, g) \\
\eta(f, g) \leq \eta(f, h)+\eta(h, g) \\
\left|\mu_{1}(f)-\mu_{1}(g)\right| \leq \varepsilon_{1}(f, g),
\end{gathered}
$$

andforf, $g \in \mathcal{P}$

$$
|f(0)-g(0)| \leq \frac{1}{2} \varepsilon_{0}(f, g) \leq \varepsilon_{1}(f, g)
$$

Proof. The inequalities (3.1)-(3.3) are obvious.

For (3.4) we have

$$
\varepsilon_{0}(f, g)-2|f(0)-g(0)|=\sum_{x=1}^{\infty}|f(x)-g(x)|-\left|\sum_{x=1}^{\infty}(f(x)-g(x))\right| \geq 0,
$$


which proves the first inequality. Furthermore,

$$
\begin{gathered}
\varepsilon_{0}(f, g)=\left|\sum_{x=1}^{\infty}(f(x)-g(x))\right|+\sum_{x=1}^{\infty}|f(x)-g(x)| \leq \\
2 \sum_{x=1}^{\infty} x|f(x)-g(x)|=2 \varepsilon_{1}(f, g),
\end{gathered}
$$

which proves the second inequality.

This completes the proof of Lemma 3.1.

Q.E.D.

\section{CONVOLUTIONS}

4A. When for $i=1, \ldots, m$ approximating $f_{i} \in \mathcal{P}$ by $g_{i} \in \mathcal{F}$, which is not necessarily in $\mathcal{P}$ itself, it is also natural to approximate the convolution $*_{i=1}^{m} f_{i}$ by $*_{i=1}^{m} g_{i}$. The convolution $h_{1} * h_{2}$ of two functions $h_{1}$ and $h_{2}$ on the non-negative integers is defined by

$$
\left(h_{1} * h_{2}\right)(x)=\sum_{y=0}^{x} h_{1}(y) h_{2}(x-y) ; \quad(x=0,1, \ldots)
$$

we also define $h^{0 *}(x)=I(x=0)$ for a function $h$ on the non-negative integers.

The following well-known properties of convolutions of distributions in $\mathcal{P}$ also hold for convolutions of functions in $\mathcal{F}$ :

$$
\begin{gathered}
h_{1} * h_{2}=h_{2} * h_{1} \\
\left(h_{1} * h_{2}\right) * h_{3}=h_{1} *\left(h_{2} * h_{3}\right) \\
h_{1} * h_{3}+h_{2} * h_{3}=\left(h_{1}+h_{2}\right) * h_{3} .
\end{gathered}
$$

Furthermore, we easily see that

$$
\begin{gathered}
\left|h_{1} * h_{2}\right| \leq\left|h_{1}\right| *\left|h_{2}\right| \\
\mu_{j}\left(h_{1}\right) \leq \mu_{j}\left(h_{2}\right) . \quad\left(h_{1} \leq h_{2} ; j=0, \quad 1\right)
\end{gathered}
$$

Lemma 4.1 If $h_{1}, h_{2} \in \mathcal{F}$ such that $\mu_{0}\left(\left|h_{i}\right|\right)<\infty$ for $\mathrm{i}=1,2$, then

$$
\mu_{0}\left(h_{1} * h_{2}\right)=\mu_{0}\left(h_{1}\right) \mu_{0}\left(h_{2}\right) \text {. }
$$


Proof. We have

$$
\begin{gathered}
\mu_{0}\left(h_{1} * h_{2}\right)=\sum_{x=0}^{\infty}\left(h_{1} * h_{2}\right)(x)=\sum_{x=0}^{\infty} \sum_{y=0}^{x} h_{1}(y) h_{2}(x-y)= \\
\sum_{y=0}^{\infty} h_{1}(y) \sum_{x=y}^{\infty} h_{2}(x-y)=\mu_{0}\left(h_{1}\right) \mu_{0}\left(h_{2}\right) .
\end{gathered}
$$

Q.E.D.

4B. We shall first consider bounds for $\varepsilon_{0}\left(*_{i=1}^{m} f_{i}, *_{i=1}^{m} g_{1}\right)$. For the proof of our main result we shall need the following lemma.

Lemma 4.2 Forf, $g, h \in \mathcal{F}$ we have

$$
\varepsilon_{0}(f * h, g * h) \leq \mu_{0}(|h|) \varepsilon_{0}(f, g) .
$$

Proof. We have

$$
\begin{gathered}
\varepsilon_{0}(f * h, g * h)=\sum_{x=0}^{\infty}|(f * h)(x)-(g * h)(x)|= \\
\sum_{x=0}^{\infty}\left|\sum_{y=0}^{x} h(y)(f(x-y)-g(x-y))\right| \leq \sum_{x=0}^{\infty} \sum_{y=0}^{x}|h(y)| f(x-y)-g(x-y) \mid= \\
\sum_{y=0}^{\infty}|h(y)| \sum_{x=y}^{\infty}|f(x-y)-g(x-y)|=\mu_{0}(|h|) \varepsilon_{0}(f, g) .
\end{gathered}
$$

Q.E.D.

Theorem 4.1 For $f_{i}, g_{i} \in \mathcal{F}(i=1, \ldots, m)$, we have

$$
\varepsilon_{0}\left(\underset{\substack{* \\ i=1}}{*} f_{i}, \underset{\substack{m \\ i=1}}{*} g_{i}\right) \leq \sum_{i=1}^{m} \varepsilon_{0}\left(f_{i}, g_{i}\right)\left(\prod_{j=1}^{i-1} \mu_{0}\left(\left|f_{j}\right|\right)\right)\left(\prod_{j=i+1}^{m} \mu_{0}\left(\left|g_{j}\right|\right)\right) \text {. }
$$

Proof. If $\mu_{0}\left(\left|f_{i}\right|\right)=\infty$ or $\mu_{0}\left(\left|g_{i}\right|\right)=\infty$ for some $i$, then the theorem obviously holds. Let us therefore assume that $\mu_{0}\left(f_{i}\right)$ and $\mu_{0}\left(g_{i}\right)$ are finite for all $i$. Under this assumption we shall prove (4.1) by induction on $m$. For $m=1$ it trivially holds. We now assume that it holds for $m=1, \ldots, n$. By using successively (3.1), Lemma 4.2 , Lemma 4.1, and (4.1), we obtain 


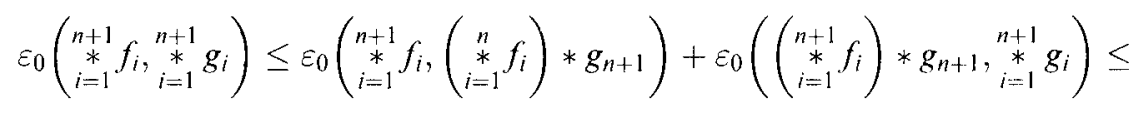

$$
\begin{aligned}
& \mu_{0}\left(\left|\underset{i}{\stackrel{n}{*} f_{i}}\right|\right) \varepsilon_{0}\left(f_{n+1}, g_{n+1}\right)+\mu_{0}\left(\left|g_{n+1}\right|\right) \varepsilon_{0}\left(\underset{i=1}{*} \underset{i}{*} f_{i}, \underset{i=1}{*} g_{i}\right) \leq \\
& \left(\prod_{i=1}^{n} \mu_{0}\left(\left|f_{i}\right|\right)\right) \varepsilon_{0}\left(f_{n+1}, g_{n+1}\right)+ \\
& \mu_{0}\left(\left|g_{n+1}\right|\right)\left\{\sum_{i=1}^{n} \varepsilon_{0}\left(f_{i}, g_{i}\right)\left(\prod_{j=1}^{i-1} \mu_{0}\left(\left|f_{j}\right|\right)\right)\left(\prod_{j=i+1}^{n} \mu_{0}\left(\left|g_{j}\right|\right)\right)\right\}= \\
& \sum_{i=1}^{n+1} \varepsilon_{0}\left(f_{i}, g_{i}\right)\left(\prod_{j=1}^{i-1} \mu_{0}\left(\left|f_{j}\right|\right)\right)\left(\prod_{j=i+1}^{n} \mu_{0}\left(\left|g_{j}\right|\right)\right)
\end{aligned}
$$

that is, (4.1) also holds for $m=n+1$. By induction it holds for all $m$. Q.E.D.

One somewhat disappointing aspect of Theorem 4.1 is that the upper bound in (4.1) is not in general invariant against permutations of the pairs $\left(f_{i}, g_{i}\right)(i=1, \ldots$, $m$ ). However, in the special case when $f_{i}, g_{i} \in \mathcal{P},(4.1)$ reduces to

$$
\varepsilon_{0}\left(\underset{i=1}{m} f_{i}, \underset{i=1}{*} g_{i}\right) \leq \sum_{i=1}^{m} \varepsilon_{0}\left(f_{i}, g_{i}\right)
$$

which is invariant.

4C. For $\eta\left(*_{i=1}^{m} f_{i}, *_{i=1}^{m} g_{i}\right)$ we have the following result.

Theorem 4:2 Forf $f_{i}, g_{i} \in \mathcal{P}(i=1, \ldots, m)$, we have

$$
\begin{aligned}
& \Pi_{*_{i=1}^{m} f} f(x)-\Pi_{\psi_{i=1}^{m}, g_{i}}(x) \leq \sum_{i=1}^{m} \sup _{y \geq 0}\left(\Pi_{f_{i}}(y)-\Pi_{g_{i}}(y)\right) \quad(x=0,1,2, \ldots) \\
& \eta\left(\underset{i=1}{*} f_{i}, \underset{i=1}{*} g_{i}\right) \leq \sum_{i=1}^{m} \eta\left(f_{i}, g_{i}\right)
\end{aligned}
$$

Proof. Formula (4.2) follows from Lemma 6 in De Pril \& Dhaene (1992), and (4.3) follows immediately from (4.2).

Q.E.D.

In (4.2) we gave an upper bound for the difference between the two stop loss transforms. By symmetry we can immediately obtain an analogous lower bound. Similarly, we shall also in the following often present our results only with upper bounds when the analogous lower bounds follow immediately by symmetry. 


\section{COMPOUND DISTRIBUTIONS}

5A. In this section we shall discuss approximations to compound distributions. For simplicity we assume that the severity distribution is in $\mathcal{P}_{+}$.

We denote the compound distribution with counting distribution $p \in \mathcal{P}$ and severity distribution $h \in \mathcal{P}_{+}$by $p \vee h$, that is,

$$
(p \vee h)(x)=\sum_{n=0}^{x} p(n) h^{n *}(x), \quad(x=0,1,2, \ldots)
$$

and we extend this definition of the function $p \vee h$ to the case when $p \in \mathcal{F}$ and $h \in \mathcal{F}_{+}$.

5B. We first consider the case when we approximate a compound distribution by approximating the counting distribution and keeping the severity distribution unchanged.

Theorem 5.1 For $p, q \in \mathcal{F}$ and $h \in \mathcal{F}_{+}$with $\mu_{0}(|h|) \leq 1$, we have

$$
\varepsilon_{0}(p \vee h, q \vee h) \leq \varepsilon_{0}(p, q)
$$

Proof. We have

$$
\begin{gathered}
\varepsilon_{0}(p \vee h, q \vee h)=\sum_{x=0}^{\infty}|(p \vee h)(x)-(q \vee h)(x)|= \\
\sum_{x=0}^{\infty}\left|\sum_{n=0}^{\infty}(p(n)-q(n)) h^{n *}(x)\right| \leq \sum_{x=0}^{\infty} \sum_{n=0}^{\infty}|p(n)-q(n)|\left|h^{n *}\right|(x)= \\
\sum_{n=0}^{\infty}|p(n)-q(n)| \sum_{x=0}^{\infty}\left|h^{n *}\right|(x)=\sum_{n=0}^{\infty}|p(n)-q(n)| \mu_{0}\left(\left|h^{n *}\right|\right) \leq \\
\sum_{n=0}^{\infty}|p(n)-q(n)| \mu_{0}^{n}(|h|) \leq \sum_{n=0}^{\infty}|p(n)-q(n)|=\varepsilon_{0}(p, q) .
\end{gathered}
$$

Q.E.D.

To deduce bounds for the approximation error for approximations to stop loss premiums, we shall need the following lemma, which is proved as formula (38) in De Pril \& Dhaene (1992).

Lemma 5.1 For $f \in \mathcal{P}$ we have

$$
n \Pi_{f}(x) \leq \Pi_{f^{n *}}(x) \leq(n-1) \mu_{1}(f)+\Pi_{f}(x) . \quad(x=0,1, \ldots ; n=1,2, \ldots)
$$

Theorem 5.2 For $h \in \mathcal{P}_{+}, p, q \in \mathcal{F}$ with $\mu_{1}(|p|)<\infty, \mu_{1}(|q|)<\infty$, and $B(p, q)=\varepsilon_{1}(p, q)-\varepsilon_{0}(p, q)+2(p(0)-q(0))_{+}+\mu_{1}(p)-\mu_{1}(q)-\mu_{0}(p)+\mu_{0}(q)$, 
we have

$$
\begin{gathered}
\Pi_{p \vee h}(x)=\Pi_{q \vee h}(x) \leq \frac{1}{2}\left(\mu_{1}(h)-\Pi_{h}(x)\right) B(p, q)+\Pi_{h}(x)\left(\mu_{1}(p)-\mu_{1}(q)\right) \\
(x=0,1,2, \ldots) \\
\eta(p \vee h, q \vee h) \leq \frac{1}{2} \mu_{1}(h)\left(\varepsilon_{1}(p, q)+\left|\mu_{1}(p)-\mu_{1}(q)\right|\right) \leq \mu_{1}(h) \varepsilon_{1}(p, q) .
\end{gathered}
$$

Proof. For $x=0,1,2, \ldots$, we have

$$
\begin{aligned}
& \Pi_{p \vee h}(x)-\Pi_{q \vee h}(x)=\sum_{y=z+1}^{\infty}(y-x)((p \vee h)(y)-(q \vee h)(y))= \\
& \sum_{y=x+1}^{\infty}(y-x) \sum_{n=1}^{\infty}(p(n)-q(n)) h^{n *}(n)=\sum_{n=1}^{\infty}(p(n)-q(n)) \Pi_{h^{n *}}(x),
\end{aligned}
$$

from which we obtain

$$
\begin{aligned}
\Pi_{p \vee h}(x)-\Pi_{q \vee h}(x) & =\sum_{n=1}^{\infty}(p(n)-q(n))\left(\Pi_{h^{\prime \prime}}(x)-n \Pi_{h}(x)\right)+ \\
& \Pi_{h}(x)\left(\mu_{1}(p)-\mu_{1}(q)\right) .
\end{aligned}
$$

Two applications of Lemma 5.1 give

$$
\begin{aligned}
& \sum_{n=1}^{\infty}(p(n)-q(n))\left(\Pi_{h^{n^{*}}}(x)-n \Pi_{h}(x)\right) \leq \\
& \sum_{n=1}^{\infty}(p(n)-q(n))_{+}\left(\Pi_{h^{n}}(x)-n \Pi_{h}(x)\right) \leq \\
& \sum_{n=1}^{\infty}(p(n)-q(n))_{+}(n-1)\left(\mu_{1}(h)-\Pi_{h}(x)\right)= \\
& \frac{1}{2}\left(\mu_{1}(h)-\Pi_{h}(x)\right) \sum_{n=1}^{\infty}(|p(n)-q(n)|+p(n)-q(n))(n-1)= \\
& \frac{1}{2}\left(\mu_{1}(h)-\Pi_{h}(x)\right) B(p, q),
\end{aligned}
$$

which together with (5.4) proves (5.2). 
As

$B(p, q)=\varepsilon_{1}(p, q)+\mu_{1}(p)-\mu_{1}(q)-2 \sum_{n=1}^{\infty}(p(n)-q(n))_{+} \leq \varepsilon_{1}(p, q)+\mu_{1}(p)-\mu_{1}(q)$,

(5.2) gives

$$
\begin{gathered}
\Pi_{p \vee h}(x)-\Pi_{q \vee h}(x) \leq \frac{1}{2}\left(\mu_{1}(h)-\Pi_{h}(x)\right)\left(\varepsilon_{1}(p, q)+\mu_{1}(p)-\mu_{1}(q)\right)+ \\
\Pi_{h}(x)\left(\mu_{1}(p)-\mu_{1}(q)\right) \leq \frac{1}{2} \mu_{1}(h)\left(\varepsilon_{1}(p, q)+\mu_{1}(p)-\mu_{1}(q)\right) .
\end{gathered}
$$

Together with the analogous inequality with interchanging of $p$ and $q$, this gives the first inequality in (5.3); the last inequality in (5.3) follows by (3.3).

This completes the proof of Theorem 5.2.

Q.E.D.

The following theorem is a special case of Theorem 1 in Sundt \& Dhaene (1996).

Theorem 5.3 For $p, q \in \mathcal{P}$ and $h \in \mathcal{P}_{+}$, we have

$$
\begin{gathered}
\Pi_{p \vee h}(x)-\Pi_{q \vee h}(x) \leq\left(\mu_{1}(h)-\Pi_{h}(x)\right) \Pi_{p}(1)+\Pi_{h}(x)\left(\mu_{1}(p)-\mu_{1}(q)\right) . \\
(x=0,1,2, \ldots)
\end{gathered}
$$

The bounds in (5.1), (5.2), and (5.3) become equal to zero when $p=q$. Unfortunately, this is not the case with the bound in (5.5) unless $\Pi_{p}(1)=0$, that is, $p$ is a Bernoulli distribution. On the other hand, we see that the bound in (5.5) is sharper than the bound in $(5.2)$ when $\Pi_{p}(1)=0$ and $p \neq q$. We shall discuss this case in more detail in subsection 6.2.

5C. Let us now consider the special case with $h \in \mathcal{P}_{+}$and $p, q \in \mathcal{P}$ with $\mu_{1}(p)=\mu_{1}(q)$. In that case (5.2), (5.5), and (5.3) reduce to respectively

$$
\begin{gathered}
\Pi_{p \vee h}(x)-\Pi_{q \vee h}(x) \leq \\
\frac{1}{2}\left(\mu_{1}(h)-\Pi_{h}(x)\right)\left(\varepsilon_{1}(p, q)-\varepsilon_{0}(p, q)+2(p(0)-q(0))_{+}\right) \quad(x=0,1,2, \ldots) \\
\Pi_{p \vee h}(x)-\Pi_{q \vee h}(x) \leq\left(\mu_{1}(h)-\Pi_{h}(x)\right) \Pi_{p}(1) \quad(x=0,1,2, \ldots) \\
\eta(p \vee h, q \vee h) \leq \frac{1}{2} \mu_{1}(h) \varepsilon_{1}(p, q) .
\end{gathered}
$$


From (5.6) we obtain

$$
\eta(p \vee h, q \vee h) \leq \frac{1}{2} \mu_{1}(h)\left(\varepsilon_{1}(p, q)-\varepsilon_{0}(p, q)+2|p(0)-q(0)|\right) .
$$

From (3.4) we see that this is sharper than or equal to the bound in (5.8).

We see that the bounds in (5.6) and (5.7) are non-decreasing in $x$. For $x=0$ these bounds become equal to zero.

5D. In subsections 5B-C we discussed approximating a compound distribution by approximating the counting distribution and keeping the severity distribution unchanged. Let us now instead consider approximating the severity distribution and keeping the counting distribution unchanged. For such approximations we have the following theorem:

Theorem 5.4 For $p \in \mathcal{F}$ and $h, k \in \mathcal{F}_{+}$with $\mu_{0}(|h|) \leq 1$ and $\mu_{0}(|k|) \leq 1$, we have

$$
\varepsilon_{0}(p \vee h, p \vee k) \leq \mu_{1}(|p|) \varepsilon_{0}(h, k) \text {. }
$$

If in addition $h, k \in \mathcal{P}_{+}$, then

$$
\eta(p \vee h, p \vee k) \leq \mu_{1}(|p|) \eta(h, k) .
$$

Proof. By application of Theorem 4.1 we obtain

$$
\begin{gathered}
\varepsilon_{0}(p \vee h, p \vee k)=\sum_{x=0}^{\infty}|(p \vee h)(x)-(p \vee k)(x)|= \\
\sum_{x=1}^{\infty}\left|\sum_{n=1}^{\infty} p(n)\left(h^{n *}(x)-k^{n *}(x)\right)\right| \leq \sum_{x=1}^{\infty} \sum_{n=1}^{\infty}|p(n)|\left|h^{n *}(x)-k^{n *}(x)\right|= \\
\sum_{n=1}^{\infty}|p(n)| \sum_{x=1}^{\infty}\left|h^{n *}(x)-k^{n *}(x)\right|=\sum_{n=1}^{\infty}|p(n)| \varepsilon_{0}\left(h^{n *}, k^{n *}\right) \leq \\
\sum_{n=1}^{\infty}|p(n)| n \varepsilon_{0}(h, k)=\mu_{1}(|p|) \varepsilon_{0}(h, k),
\end{gathered}
$$

which proves (5.10).

We now assume that $h, k \in \mathcal{P}_{+}$. For $x=0,1,2, \ldots$ we obtain

$$
\begin{gathered}
\left|\Pi_{p \vee h}(x)-\Pi_{p \vee k}(x)\right|=\left|\sum_{n=1}^{\infty} p(n)\left(\Pi_{k^{n *}}(x)-\Pi_{k^{n *}}(x)\right)\right| \leq \\
\sum_{n=1}^{\infty}|p(n)|\left|\Pi_{h^{n *}}(x)-\Pi_{k^{\prime *}}(x)\right| \leq \sum_{n=1}^{\infty}|p(n)| \eta\left(h^{n *}, k^{n *}\right) .
\end{gathered}
$$


Application of (4.3) gives

$$
\left|\Pi_{p \vee h}(x)-\Pi_{p \vee k}(x)\right| \leq \sum_{n=1}^{\infty}|p(n)| n \eta(h, k)=\mu_{1}(|p|) \eta(h, k),
$$

from which we obtain (5.11).

This completes the proof of Theorem 5.3.

Q.E.D.

5E. We shall now discuss two classes of approximations that can be convenient both for the counting distribution and the severity distribution in a compound distribution.

For $f \in \mathcal{P}$ we define the approximation $f^{(r)}$ for a positive integer $r$ by

$$
f^{(r)}(x)=f(x) I(x \leq r) . \quad(x=0,1,2, \ldots)
$$

As

$$
\mu_{j}\left(f^{(r)}\right)=\sum_{x=0}^{r} x^{j} f(x) \quad \varepsilon_{j}\left(f, f^{(r)}\right)=\sum_{x=r+1}^{\infty} x^{j} f(x), \quad(j=0,1)
$$

we obtain

$$
\begin{gathered}
\varepsilon_{j}\left(f, f^{(r)}\right)+\mu_{j}\left(f^{(r)}\right)=\mu_{j}(f) \quad(j=0,1) \\
\mu_{0}\left(f^{(r)}\right)=\Gamma_{f}(r) \\
\varepsilon_{1}\left(f, f^{(r)}\right)=\Pi_{f}(r)+r\left(1-\Gamma_{f}(r)\right) .
\end{gathered}
$$

As $f(x) \geq f^{(r)}(x)$ for $x=0,1,2, \ldots, \Pi_{f}(x)-\Pi_{f^{(r)}}(x)$ is non-negative and nonincreasing in $x$, and we obtain

$$
\eta\left(f, f^{(r)}\right)=\Pi_{f}(r)-\Pi_{f^{(r)}}(0)=\mu_{1}(f)-\mu_{1}\left(f^{(r)}\right)=\varepsilon_{1}\left(f, f^{(r)}\right) .
$$

We see that unless $\Gamma_{f}(r)=1$, the approximation $f^{(r)}$ will not be a proper distribution as $\mu_{0}\left(f^{(r)}\right)<\mu_{0}(f)=1$. To obtain a proper distribution, we can apply the modified approximation $\tilde{f}^{(r)}$ defined by

$$
\tilde{f}^{(r)}(x)= \begin{cases}f(x) & (x=0,1, \ldots, r-1) \\ 1-\Gamma_{f}(r-1) & (x=r) \\ 0 . & (x=r+1, r+2, \ldots)\end{cases}
$$

For $j=0,1$ we get

$$
\begin{gathered}
\mu_{j}\left(\tilde{f}^{(r)}\right)=\mu_{j}\left(f^{(r)}\right)+r^{j}\left(\mathbf{I}-\Gamma_{f}(r)\right) \\
\varepsilon_{j}\left(f, \tilde{f}^{(r)}\right)=\varepsilon_{j}\left(f, f^{(r)}\right)+r^{j}\left(1-\Gamma_{f}(r)\right) .
\end{gathered}
$$


It is easily shown that

$$
\Pi_{f}(x)-\Pi_{f^{(r)}}(x)=\Pi_{f}(\max (x, r)), \quad(x=0,1,2, \ldots)
$$

from which we obtain

$$
\eta\left(f, \tilde{f}^{(r)}\right)=\Pi_{f}(r)
$$

If $X$ is a random variable with distribution $f$, then $\tilde{f}^{(r)}$ is the distribution of $\tilde{X}^{(r)}=\min (X, r)$. As $\tilde{X}^{(r)} \leq X$, we immediately obtain inequalities like

$$
\Gamma_{\tilde{f}^{(r)}}(x) \geq \Gamma_{f}(x) \quad \Pi_{f^{(n)}}(x) \leq \Pi_{f}(x) . \quad(x=0,1,2, \ldots)
$$

Theorem 5.5 If $p, h \in \mathcal{P}$ and $r$ and $x$ are positive integers, then

$$
\begin{aligned}
& 0 \leq \Pi_{p \vee h}(x)-\Pi_{\bar{p}^{(r) \vee h}}(x) \leq \mu_{1}(h) \Pi_{p}(r) \\
& 0 \leq \Pi_{p \vee h}(x)-\Pi_{p \vee \tilde{h}^{(r)}}(x) \leq \Pi_{h}(r) \mu_{1}(p) .
\end{aligned}
$$

Proof. Sundt (1991) proved (5.15). The last inequality in (5.16) follows from Theorem 5.4 and (5.14), and the first inequality is immediately seen by interpreting $\Pi_{p \vee h}(x)-\Pi_{p \vee \tilde{h}^{(r)}}(x)$ as the mean of a non-negative random variable.

This completes the proof of Theorem 5.5.

Q.E.D.

We notice that

$$
\begin{gathered}
\varepsilon_{j}\left(f, \tilde{f}^{(r)}\right) \geq \varepsilon_{j}\left(f, f^{(r)}\right) \quad(j=0,1) \\
\eta\left(f, \tilde{f}^{(r)}\right) \leq \eta\left(f, f^{(r)}\right) .
\end{gathered}
$$

5F. By combining the results from Section 5 with the results from Section 4, we can obtain error bounds for approximations to convolutions of compound distributions. For a simple illustration, let $p_{i} \in \mathcal{P}$ and $h_{i} \in \mathcal{P}_{+}(i=1, \ldots, m)$. From Theorem 4.1, (5.1), (5.12), and (5.13), we obtain

$$
\begin{gathered}
\varepsilon_{0}\left(\underset{i=1}{*} \underset{i=1}{*}\left(p_{i} \vee h_{i}\right), \underset{i=1}{*} \underset{i}{*}\left(p_{i}^{(r)} \vee h_{i}\right)\right) \leq \sum_{i=1}^{m} \varepsilon_{0}\left(p_{i} \vee h_{i}, p_{i}^{(r)} \vee h_{i}\right) \leq \\
\sum_{i=1}^{m} \varepsilon_{0}\left(p_{i}, p_{i}^{(r)}\right)=\sum_{i=1}^{m}\left(1-\Gamma_{p_{i}}(r)\right) .
\end{gathered}
$$




\section{APPLICATIONS}

\subsection{Introduction}

In this section we shall under various assumptions discuss approximations to compound distributions by approximating the counting distribution with another distribution with the same mean and keeping the severity distribution fixed, that is, we want to approximate $p \vee h$ with $q \vee h$ when $p, q \in \mathcal{P}, h \in \mathcal{P}_{+}$and $\mu_{1}(q)=\mu_{1}(p)$.

\subsection{Bernoulli distribution}

Lemma 6.1 If $p$ is a Bernoulli distribution and $q \in \mathcal{P}$ with $\mu_{1}(q)=\mu_{1}(p)$, then

$$
\begin{gathered}
q(0) \geq p(0) \quad q(1) \leq p(1) \\
\varepsilon_{0}(p, q)=\varepsilon_{1}(p, q)=2(p(1)-q(1)) \\
\Pi_{p}(1)=0 \\
\Pi_{q}(1)=q(0)-p(0) .
\end{gathered}
$$

Proof. We have

$$
1-p(0)=p(1)=\mu_{1}(p)=\mu_{1}(q)=\sum_{n=1}^{\infty} n q(n) \geq \sum_{n=1}^{\infty} q(n)=1-q(0) \geq q(1),
$$

which proves $(6.1)$.

We have

$$
\begin{gathered}
\varepsilon_{0}(p, q)=\sum_{n=0}^{\infty}|p(n)-q(n)|=q(0)-p(0)+p(1)-q(1)+\sum_{n=2}^{\infty} q(n)= \\
2(p(1)-q(1)) \\
\varepsilon_{1}(p, q)=\sum_{n=1}^{\infty} n|p(n)-q(n)|=p(1)-q(1)+\sum_{n=2}^{\infty} n q(n)= \\
p(1)-q(1)+\mu_{1}(q)-q(1)=2(p(1)-q(1)),
\end{gathered}
$$

which prove (6.2). 
Formula (6.3) is obvious.

We have

$$
\Pi_{q}(1)=\sum_{n=1}^{\infty}(n-1) q(n)=\mu_{1}(q)-(1-q(0))=p(1)-1+q(0)=q(0)-p(0),
$$

which proves $(6.4)$.

This completes the proof of Lemma 6.1.

Q.E.D.

By application of (6.2) to respectively (5.1) and (5.9), we obtain

$$
\begin{gathered}
\varepsilon_{0}(p \vee h, q \vee h) \leq 2(p(1)-q(1)) \\
\eta(p \vee h, q \vee h) \leq \mu_{1}(h)(q(0)-p(0)),
\end{gathered}
$$

and insertion of (6.3) and (6.4) in (5.7) gives

$$
-\left(\mu_{1}(h)-\Pi_{h}(x)\right)(q(0)-p(0)) \leq \Pi_{p \vee / h}(x)-\Pi_{q \vee h}(x) \leq 0 \quad(x=0,1,2, \ldots)
$$

the second inequality was proved by Bühlmann et al. (1977).

\subsection{Binomial distribution}

We now assume that

$$
p(n)=\left(\begin{array}{c}
t \\
n
\end{array}\right) \pi^{n}(1-\pi)^{t-n} . \quad(n=0,1, \ldots, t ; t=1,2, \ldots ; 0<\pi<1)
$$

The Bernoutli distribution discussed in subsection 6.2 occurs as a special case with $t=1$. However, unfortunately the situation becomes more complicated when $t>1$.

In the general case we have

$$
\begin{gathered}
\mu_{1}(p)=t \pi \\
\Pi_{p}(1)=t \pi+(1-\pi)^{t}-1 \quad \Pi_{q}(1)=t \pi+q(0)-1,
\end{gathered}
$$

and insertion in (5.7) gives

$$
\begin{aligned}
& -\left(\mu_{1}(h)-\Pi_{h}(x)\right)(t \pi+q(0)-1) \leq \Pi_{p \vee h}(x)-\Pi_{q \vee h}(x) \leq \\
& \left(\mu_{1}(h)-\Pi_{h}(x)\right)\left(t \pi-(1-\pi)^{\prime}-1\right) . \quad(x=0,1,2, \ldots)
\end{aligned}
$$


Unfortunately, when $t>1$, the upper bound does not become equal to zero like in the case $t=1$. However, as the present binomial distribution is the $t$-fold convolution of the Bernoulli distribution $p_{t}$ given by

$$
p_{t}(1)=1-p_{i}(0)=\pi
$$

it is tempting to apply the results of Theorems 4.1 and 4.2. To be able to do that, we have to assume that there exists a distribution $q_{t} \in \mathcal{P}$ such that $q=q_{t}^{* *}$. Under this assumption we have

$$
p \vee h=\left(p_{t} \vee h\right)^{t *} \quad q \vee h=\left(q_{t} \vee h\right)^{t *} .
$$

From Theorem 4.1 and (6.5) we obtain

$$
\varepsilon_{0}(p \vee h, q \vee h) \leq t \varepsilon_{0}\left(p_{t} \vee h, q_{t} \vee h\right) \leq 2 t\left(\pi-q_{t}(1)\right) .
$$

We obviously have

$$
\begin{gathered}
q(0)=q_{t}(0)^{t} \\
q(1)=t q_{t}(0)^{t-1} q_{t}(1) .
\end{gathered}
$$

Thus

$$
q_{t}(1)=\frac{1}{t} \frac{q(1)}{q(0)} q(0)^{\frac{1}{1}}
$$

and insertion in (6.11) gives

$$
\varepsilon_{0}(p \vee h, q \vee h) \leq 2\left(t \pi-\frac{q(1)}{q(0)} q(0)^{\frac{1}{1}}\right)
$$

From Theorem 4.2, (6.7), and (6.10) we obtain

$$
\begin{array}{r}
-\left(\mu_{1}(h)-\Pi_{h}(x)\right)(t \pi+q(0)-1) \leq \Pi_{p \vee h}(x)-\Pi_{q \vee h}(x) \leq 0, \\
(x=0,1,2, \ldots)
\end{array}
$$

which implies

$$
\eta(p \vee h, q \vee h) \leq \mu_{1}(h)(t \pi+q(0)-1)
$$

However, from Theorem 4.2, (6.6), and (6.12) we obtain

$$
\eta(p \vee h, q \vee h) \leq t \mu_{1}(h)\left(\pi+q(0)^{\frac{1}{t}}-1\right)
$$

which gives a sharper bound when $t>1$. This implies that the lower bound in (6.14) is sharper than the bound in (6.15) only for high values for $\Pi_{h}(x)$, that is, low values of $x$. 
The distribution $q$ is called infinitely divisible if there for each positive integer $m$ exists a distribution $q_{m}$ such that $q=q_{m}^{m *}$ (cf. e.g. Feller (1968)). In particular, this condition should hold for $m=t$, and thus (6.13)-(6.15) hold when $q$ is infinitely divisible.

The condition that there has to exist a distribution $q_{t}$ such that $q=q_{1}^{i *}$, may seem intuitively unnatural. However, the following example shows that the inequality $\Pi_{q \vee h} \leq \Pi_{p \vee h}$ does not necessarily hold when this condition is not fulfilled.

Example. Let $t=2, \pi=\frac{1}{2}$, and

$$
q(0)=q(2)=\frac{1}{8} \quad q(1)=\frac{3}{4} .
$$

Then $\mu_{1}(p)=\mu_{1}(q)=1$, and application of (6.9) gives $\Pi_{p}(\mathbf{1})-\Pi_{q}(\mathbf{1})=\frac{1}{8}>0$.

\subsection{Two infinitely divisible distributions}

We shall now assume that both $p$ and $q$ are infinitely divisible. From Theorem $4.2,(5.7)$, and (6.12) we obtain that for each positive integer $m$

$$
\begin{gathered}
-\mu_{1}(h)\left(\mu_{1}(p)+m q(0)^{\frac{1}{m}}-m\right) \leq \Pi_{p \vee h}(x)-\Pi_{q \vee h}(x) \leq \\
\mu_{1}(h)\left(\mu_{1}(p)+m p(0)^{\frac{1}{m}}-m\right), \quad(x=0,1,2, \ldots)
\end{gathered}
$$

and by letting $m$ go to infinity we obtain

$$
\begin{gathered}
-\mu_{1}(h)\left(\mu_{1}(p)+\ln q(0)\right) \leq \Pi_{p \vee h}(x)-\Pi_{q \vee h}(x) \leq \\
\mu_{1}(h)\left(\mu_{1}(p)+\ln p(0)\right) . \quad(x=0,1,2, \ldots)
\end{gathered}
$$

\subsection{Poisson vs. infinitelv divisible distribution}

We now assume that

$$
p(n)=\frac{\lambda^{n}}{n} e^{-\lambda}, \quad(n=0,1,2, \ldots ; \lambda>0)
$$

and that $q$ is infinitely divisible. Then $p$ is also infinitely divisible, and we have $\mu_{1}(p)=\lambda$. 
Let

$$
\bar{p}_{t}(n)=\left(\begin{array}{c}
t \\
n
\end{array}\right)\left(\frac{\lambda}{t}\right)^{n}\left(1-\frac{\lambda}{t}\right)^{t-n} . \quad(n=0,1, \ldots, m ; t=1,2, \ldots)
$$

Then

$$
p(n)=\lim _{t \uparrow \infty} \bar{p}_{t}(n) . \quad(n=0,1,2, \ldots)
$$

From (6.13) we obtain

$$
\varepsilon_{0}\left(\bar{p}_{t} \vee h, q \vee h\right) \leq 2\left(\lambda-\frac{q(1)}{q(0)} q(0)^{\frac{1}{\iota}}\right),
$$

and as this bound is decreasing in $t$, we obtain

$$
\varepsilon_{0}(p \vee h, q \vee h) \leq 2\left(\lambda-\frac{q(1)}{q(0)}\right)
$$

by letting $t$ go to infinity. A similar limiting argument for (6.14) gives

$$
-\left(\mu_{1}(h)-\Pi_{h}(x)\right)(\lambda+q(0)-1) \leq \Pi_{p \vee h}(x)-\Pi_{q \vee h}(x) \leq 0 . \quad(x=0,1,2, \ldots)
$$

From (6.16) and (6.19) we obtain

$$
\eta(p \vee h, q \vee h) \leq \mu_{1}(h)(\lambda+\ln q(0)),
$$

which could also have been found by a limiting argument in (6.15). As ln $q(0) \leq q(0)-1$, the lower bound in (6.19) in weaker than (6.20) for large values of $x$.

\subsection{Binomial vs. negative binomial distribution}

We now assume that $p$ is the binomial distribution given by (6.8), and that $q$ is given by

$$
q(n)=\left(\begin{array}{c}
\alpha+n-1 \\
n
\end{array}\right)(1-\rho)^{\alpha} \rho^{n} . \quad(n=0,1, \ldots ; \alpha>0 ; 0<\rho<1)
$$

Then $q$ is infinitely divisible with

$$
\mu_{1}(q)=\alpha \frac{\rho}{1-\rho}
$$

and from (6.13)-(6.15) we obtain 


$$
\begin{gathered}
\varepsilon_{0}(p \vee h, q \vee h) \leq 2 t \pi\left(1-(1-\rho)^{\frac{x+1}{t}+1}\right) \\
-\left(\mu_{1}(h)-\Pi_{h}(x)\right)\left[t \pi+(1-\rho)^{\alpha}-1\right] \leq \Pi_{p \vee h}(x)-\Pi_{q \vee h}(x) \leq 0 \\
(x=0,1,2, \ldots) \\
\eta(p \vee h, q \vee h) \leq t \mu_{1}(h)\left(\pi+(1-\rho)^{\frac{a}{t}}-1\right) .
\end{gathered}
$$

\subsection{Binomial vs. Poisson distribution}

We now assume that $p$ is the binomial distribution given by (6.8) and $q$ the Poisson distribution given by (6.17). Then (6.13)-(6.15) give

$$
\begin{gathered}
\varepsilon_{0}(p \vee h, q \vee h) \leq 2 t \pi\left(1-e^{-\pi}\right) \\
-\left(\mu_{1}(h)-\Pi_{h}(x)\right)\left(t \pi+e^{-t \pi}-1\right) \leq \Pi_{p \vee h}(x)-\Pi_{q \vee h}(x) \leq 0 \\
(x=0,1,2, \ldots) \\
\eta(p \vee h, q \vee h) \leq t \mu_{1}(h)\left(\pi+e^{-\pi}-1\right),
\end{gathered}
$$

which can also be deduced from (6.22)-(6.24) by a limiting argument.

\subsection{Poisson vs. negative binomial distribution}

We now assume that $p$ is the Poisson distribution given by (6.17) and $q$ the negative binomial distribution given by (6.21). Then (6.18)-(6.20) give

$$
\begin{gathered}
\varepsilon_{0}(p \vee h, q \vee h) \leq 2 \alpha \frac{\rho^{2}}{1-\rho} \\
-\left(\mu_{1}(h)-\Pi_{h}(x)\right)\left(\alpha \frac{\rho}{1-\rho}+(1-\rho)^{\alpha}-1\right) \leq \Pi_{p \vee h}(x)-\Pi_{q \vee h}(x) \leq 0 \\
(x=0,1,2, \ldots) \\
\eta(p \vee h, q \vee h) \leq \alpha \mu_{1}(h)\left(\frac{\rho}{1-\rho}+\ln (1-\rho)\right),
\end{gathered}
$$

which can also be deduced from (6.22)-(6.24) by a limiting argument.

The bound in (6.28) was deduced by Gerber (1984) and the bound in (6.30) by Dhaene (1991). 


\subsection{Collective approximation to individual model}

For $i=1, \ldots, m$, let $h_{i} \in \mathcal{P}_{+}$and $p_{i}$ be the Bernoulli distribution given by

$$
p_{i}(\mathbf{1})=1-p_{i}(\mathbf{0})=\pi_{i} \text {. }
$$

We approximate $p_{i} \vee h_{i}$ with the compound Poisson distribution $q_{i} \vee h_{i}$ with

$$
q_{i}(n)=\frac{\pi_{i}^{n}}{n !} e^{-\pi_{i}} . \quad(n=0,1,2, \ldots)
$$

It is well known that then $*_{i=1}^{m}\left(q_{i} \vee h_{i}\right)=q \vee h$ with

$$
\begin{aligned}
& q(n)=\frac{\lambda^{n}}{n} e^{-\lambda} \quad(n=0,1,2, \ldots) \\
& \lambda=\sum_{i=1}^{m} \pi_{i} \quad h=\frac{1}{\lambda} \sum_{i=1}^{m} \pi_{i} h_{i} .
\end{aligned}
$$

By a trivial generalisation of (6.25) and (6.27) we obtain

$$
\begin{aligned}
& \varepsilon_{0}\left(\underset{\substack{* \\
i=1}}{*}\left(p_{i} \vee h_{i}\right), q \vee h\right) \leq 2 \sum_{i=1}^{m} \pi_{i}\left(1-e^{-\pi_{i}}\right)
\end{aligned}
$$

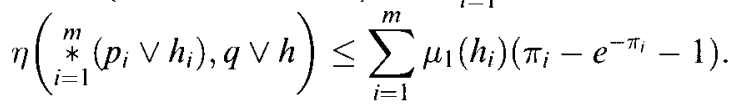

Unfortunately we have not been able to generalise the first inequality in (6.26), but the second inequality is easily generalised to

$$
\Pi_{*_{i, 1}^{\prime \prime},\left(p_{i} \vee h_{i}\right)}(x) \leq \Pi_{q \vee h}(x) . \quad(x=0,1,2, \ldots)
$$

The inequalities in (6.31)-(6.33) have been deduced by respectively Gerber (1984), De Pril \& Dhaene (1992), and Bühlmann et al. (1977).

When $\pi_{i}$ and $h_{i}$ are the same for all $i$, we are back in the situation of subsection 6.7 .

\section{REFERENCES}

Bühlmann, H., Gagliardi, B., Gerber, H.U., \& Straub, E. (1977). Some inequalities for stop-loss premiums. ASTIN Bulletin 9, 75-83.

De Pril, N. \& Dhaene, J. (1992). Error bounds for compound Poisson approximations to the individual risk model. ASTIN Bulletin 22, 135-148.

DHAENe, J. (1991). Approximating the compound negative binomial distribution by the compound Poisson distribution. Bulletin of the Swiss Association of Actuaries, 117-121.

DHAENE, J. \& SUNDT, B. (1996). On approximating distributions by approximating their De Pril transforms. To be published in Scandinavian Actuarial Journal.

FELLER, W, (1968). An introduction to probability theory and its applications. (3. ed.). Wiley. New York.

Gerber, H.U. (1984). Error bounds for the compound Poisson approximation. Insurance: Mathematics and Economics 3, 191-194. 
Sundt, B. (1991). On approximating aggregate claims distributions and stop-loss premiums by truncation. Insurance: Mathematics and Economics 10, 133-136

Sundt, B. \& DhaEne, J. (1996). On bounds for the difference between the stop loss transforms of two compound distributions. ASTIN Bulletin 26, 225-231.

JAN DHAENE

Departement Toegepaste Economische

Wetenschappen

Katholieke Universiteit Leuven

Huis Eighen Heerd

Minderbroederstraat 5

$B$-3000 Leuven

Belgium
BJøRN SUNDT

Department of Mathematics

University of Bergen

Johannes Brunsgate 12

N-5008 Bergen

Norway 